\title{
GASTROMEGALY IN CHILDREN
}

\author{
BY \\ HUGH G. GARLAND, M.D., M.R.C.P., \\ AND \\ B. S. PLATT, M.B., M.Sc., Ph.D. \\ (From the Children's Department, General Infirmary, Leeds.)
}

Gastromegaly associated with chronic duodenal ileus has been described for nearly a century and though there is now a considerable literature on the subject it is still in some countries largely unrecognized. In France it is considered to be a common and important cause of dyspepsia, but it receives scant attention in this country.

The causes, and suggested causes, of chronic duodenal ileus are numerous. They vary from congenital megaduodenum and obstruction by a congenital abnormality in the arterio-mesenteric band (the root of the mesentery carrying the superior mesenteric vessels), to autonomic imbalance; but probably congenital and inflammatory bands constitute the commonest causes in children. Most of the English literature on duodenal stasis comes from Wilkie ${ }^{1}$ in the case of adults, and from Miller and $\mathrm{Gage}^{2,3}$ who have made an extensive survey of recorded cases in children. The latter authors first drew attention to the association of gastromegaly and chronic duodenal ileus in 1930, following this up with a further communication in 1932. They consider that an accurate clinical diagnosis of the condition is possible and stress the striking improvement of these cases under medical treatment; they consider arterio-mesenteric obstruction to be the most important factor.

The clinical picture painted by Miller and Gage is as follows. The child shows refusal of food from an early age together with vomiting, often forcible in type; the vomitus may or may not be bile-stained and it contains excess of mucus. Vomiting becomes more troublesome in the second year and tends to occur in attacks with pyrexia, prostration and diarrhœa; there is frequently anorexia between attacks. Physical development is retarded, though stunting in height is not a prominent feature; the abdomen is protuberant and there may be visible gastric peristalsis. Radioscopy shows a large stomach with delayed emptying and a well-filled duodenal cap. The condition improves considerably when treated with frequent, small, dry meals together with alkalies, and it tends to disappear spontaneously about the fifth year.

We record here two cases which have been under the care of Dr. C. W. Vining. One of them died, and the other showed striking improvement under medical treatment. 
Case 1.-Maurice S., a full-time baby, was breast-fed for 9 months. Frequent, forcible vomiting occurred from birth, immediately or shortly after food, the vomit being usually yellow and presumably bile-stained. Associated with the vomiting were attacks of abdominal pain with drawing-up of the legs, and occasionally there were attacks of diarrhœa with slimy stools alternating with attacks of severe constipation. In spite of these disturbances the child was robust and gaining in weight. In November, 1928, he was admitted to the surgical department of the Leeds General Infirmary on account of abdominal pain, vomiting and tetany, but apart from some evidence of rickets examination was negative. From this time onwards he had

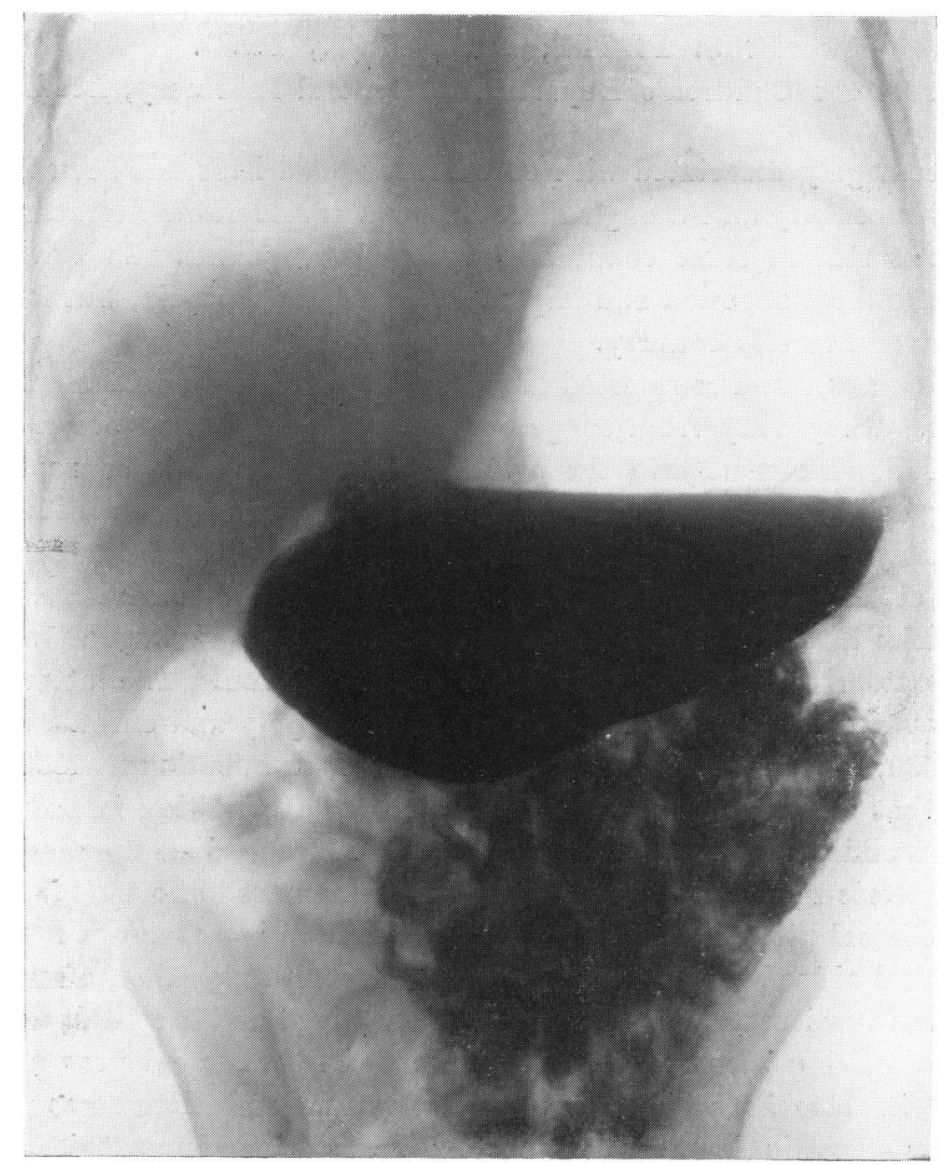

attacks of vomiting of increasing frequency, which were preceded by flushing of the face and accompanied by pyrexia. An attack of vomiting began on November 5th, 1930, and tetany developed within twenty-four hours, accompanied by hiccough, a feature emphasized by Miller and Gage. As the vomiting continued for two days he was admitted to the Leeds General Infirmary where he was examined by one of us (B.S.P.). There was marked cyanosis, the pulse being rapid and feeble. Carpopedal spasm affected the two sides of the body alternately and Chvostek's sign was present. Continuous movenents of a fibrillating nature were felt in the epigastrium. The tetany and cyanosis were relieved by the administration of a mixture of oxygen 


\section{GASTROMEGALY}

with $\mathrm{CO}_{2}$ (5 per cent.), together with parathormone; but in spite of this the patient died suddenly two hours after admission.

An autopsy was carried out the following day by one of us (H.G.G.). Some degree of carpo-pedal spasm remained. On opening the abdomen the stomach was found to be injected, enormously dilated and contained a large quantity of bilestained food mixed with excess of mucus. The mucosa appeared normal and there was no pyloric obstruction. The duodenal mucosa was bile-staired; the greater part of the duodenum was greatly dilated and approximately at the duodeno-jejunal

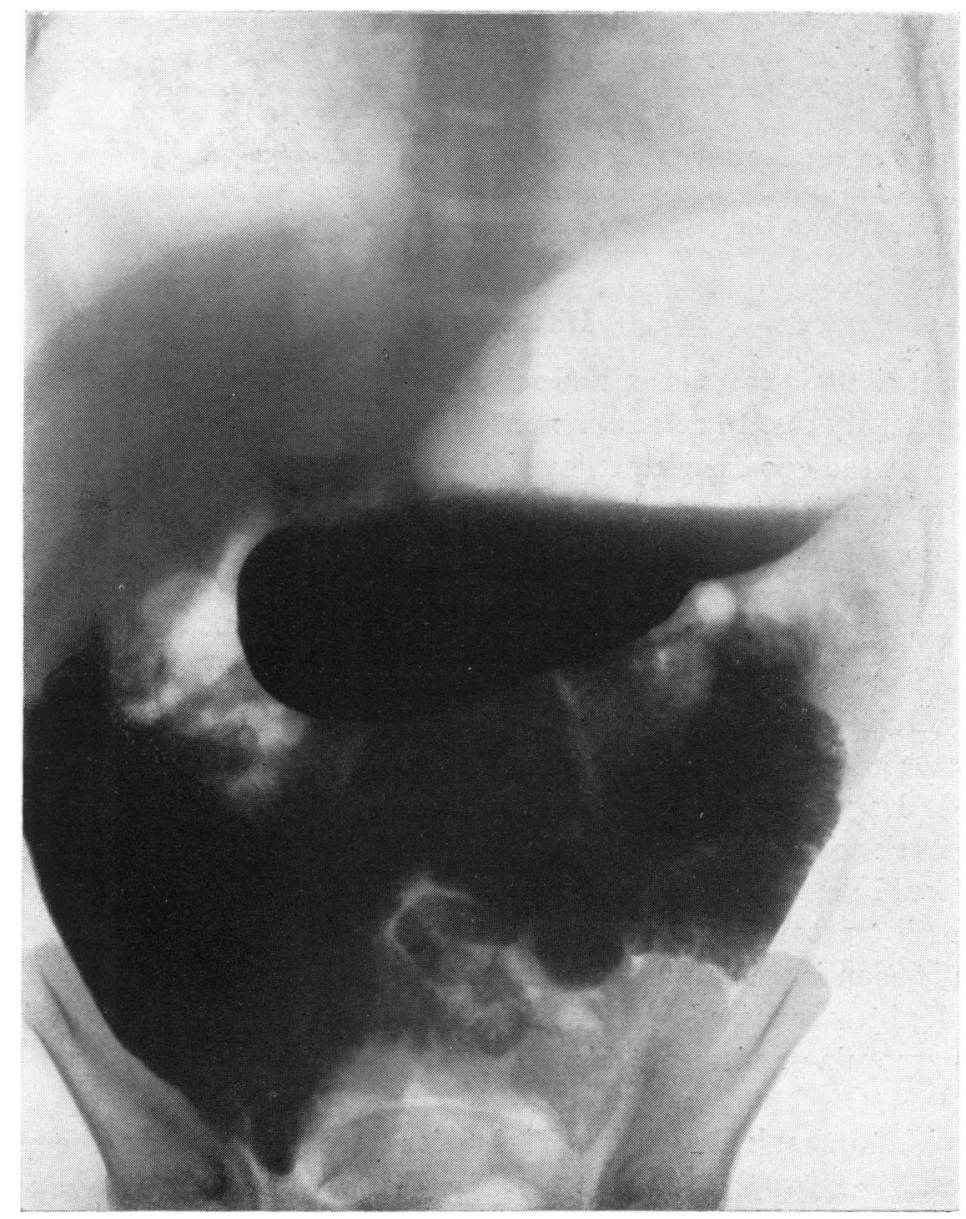

junction there was an incomplete obstruction of the lumen which was easily overcome by a current of water. This obstruction was due to a tight band formed by the root of the mesentery and which, on section, was found to contain the superior mesenteric vessels. Distal to the obstruction the small intestine was collapsed and injected; the colon appeared normal. The spleen showed a few subcapsular hæmorrhages and all the other organs were normal to the naked-eye.

Case 2. -Stewart, H., was first seen in January, 1931, when he was nearly two years old. He was a full-time, breast-fed baby. From the seventh month onwards 
he had been subject to attacks of an indefinite nature and described by the parents as being like 'a faint without loss of consciousness'; he became pale and suffered from profuse diarrhœa and later began to vomit. He had had four such attacks at intervals of some months, but at more frequent intervals there had been attacks of less severe diarrhœa and vomiting, alternating with periods of constipation. He failed to put on weight and frequently complained of stomach ache.

On examination he was found to be underweight, but apart from a rather protuberant abdomen nothing abnormal was discovered. X-ray examination showed marked dilatation of the stomach (Fig. 1) with considerable delay in emptying. A provisional diagnosis of chronic duodenal ileus was made and he was treated with alkalies and given small, dry meals. He was re-examined after 18 months in August, 1932, when he shewed marked improvement; he had gained weight, the stools were quite normal, there had been no further vomiting and the child looked well. He was X-rayed for the second time in September, 1932, when the stomach was still seen to be dilated but to less an extent (Fig. 2). The improvement in health has been maintained up to the present time.

\section{Discussion.}

The first of these cases is an undoubted example of chronic duodenal ileus and gastromegaly due to arterio-mesenteric obstruction. The case is striking on account of the good health enjoyed by the child between the attacks and for the fatal termination. The second case we consider to be an example of the same condition which is responding well to medical treatment.

Gastromegaly due to duodenal stasis is an important and probably fairly common condition in children which should be treated medically, but if the attacks fail to clear up under such treatment duodeno-jejunostomy is probably advisable. The success of medical treatment apparently depends on the prevention of dilatation of the stomach by large meals, as the downward drag increases the duodenal obstruction. In the event of a severe attack of vomiting gastric lavage should be carried out and tetany should be treated with parathormone; if the attack persists in spite of such treatment operation may have to be undertaken at once. A fatal termination is probably unusual, but the condition is serious, and many cases no doubt masquerade under such titles as cyclical vomiting, migraine, nervous dyspepsia or visceroptosis.

We wish to thank Dr. C. W. Vining for permission to use the notes of these cases.

\section{REFERENGES.}

1. Wilkie, D. P. D., Am. J. Med. Sc., Philad., 1927, CLXXIII, 643.

2. Miller, R., \& Gage, H. C., Arch. Dis. Child., London, 1930, V, 83.

3. , ,,$\quad$ Ibid., 1932, VII, 65. 